\title{
Rapid determination of colloidal crystal's structure by reflection spectrum
}

\author{
Hongwei Zhou ${ }^{\mathrm{a}, \mathrm{b}}$, Shenghua Xu ${ }^{\mathrm{a}, \mathrm{b}}$, Zhiwei Sun ${ }^{\mathrm{a}, \mathrm{b}, *}, \mathrm{Xuan}_{\mathrm{Du}}^{\mathrm{a}, \mathrm{b}}$, Jingchang Xie ${ }^{\mathrm{a}, \mathrm{b}}$ \\ a Key Laboratory of Microgravity, Institute of Mechanics, Chinese Academy of Sciences, No. 15 Beisihuanxi Road, Beijing 100190, People's Republic of China \\ ${ }^{\mathrm{b}}$ National Microgravity Laboratory, Institute of Mechanics, Chinese Academy of Sciences, No. 15 Beisihuanxi Road, Beijing 100190, People's Republic of China
}

\section{A R T I C L E I N F O}

\section{Article history}

Received 3 September 2010

Received in revised form

10 November 2010

Accepted 18 November 2010

Available online 26 November 2010

\section{Keywords:}

Colloidal crystal

Crystal structure

Reflection spectrum

Static light scattering

\begin{abstract}
A B S T R A C T
We prove that the reflection spectrum (RS), which was thought to be not able to determine the crystal structure, can be used to determine the colloidal crystal's structure with a rather fast sample rate. This method shows its superiority by comparing with conventional static light scattering (SLS) method. The result greatly extended the applicability of the reflection spectrum method, which has already been used in measuring lattice spacing and crystal size, etc. in the study of colloidal crystals. Therefore, it becomes possible for the reflection spectrum method to simultaneously and rapidly determine the crystal structure, lattice constant and crystal size, etc. of colloidal crystals, which is essential for deep understanding of phase behavior and crystallization kinetics.
\end{abstract}

(c) 2010 Elsevier B.V. All rights reserved.

\section{Introduction}

Colloidal suspensions have acquired a model system status in probing the underlying physics in condensed matters because of the analogy between atomic and colloidal system. Larger particle size and slower dynamics greatly facilitate the study both in temporal scale and spatial scale. Rich phase behaviors including disorder-order-reentrant fluid [1-4] and phase diagrams [5-7] have been observed experimentally for suspensions of charged colloidal particles. Conventionally it was believed that the charged colloidal particles interact predominantly via a screened Coulomb repulsion according to DLVO (Derjaguin-Landau-Verwey-Overbeek) theory. But recent studies have shown that attractive interparticle interaction could exist between highly charged particles [8-10]. The range and strength of interparticle interaction potential can be varied over a wide range by changing the particle concentration, foreign salt concentration and surface charge numbers. By controlling these parameters, different crystallization behaviors can be observed.

The crystal structure, lattice constant and crystal size, etc., are important parameters that describe the characteristics of crystals. Therefore, the rapid measurement of these parameters during crystallization is of great importance, especially for studying the crystallization kinetics. On the other hand, the simultaneous deter-

\footnotetext{
* Corresponding author at: Key Laboratory of Microgravity, Institute of Mechanics, Chinese Academy of Sciences, No. 15 Beisihuanxi Road, Beijing 100190, People's Republic of China. Tel.: +8610 82544093; fax: +8610 82544096 .

E-mail address: sunzw@imech.ac.cn (Z. Sun).
}

mination of these parameters is also necessary, because these parameters might have some relationship during the crystallization process. Normally, different methods on studying these parameters of colloidal crystals may have different advantages. However most of the present methods cannot satisfy both the rapid and the simultaneous measurement.

Reflection spectrum can achieve a fast sampling rate easily and has been used in the study of nucleation and growth velocity of colloidal crystal, in which the lattice spacing and crystal size were measured $[11,12]$. However, according to the present viewpoints, the crystal structure can not be determined by the reflection spectrum method alone $[13,14]$.

At present, the mainly used methods to analyze the crystal structure are Kossel line [15-17], static light scattering (SLS) [18-21] and ultra-small-angle X-ray scattering (USAXS) [22-25]. Kossel line can be used to determine crystal structure and lattice constant with high accuracy, however sometimes a moderate degree of perfection of the crystallites and certain grain sizes are needed [15]. SLS is another very effectively and widely used method which can characterize the colloidal crystal's structure. It is highly analogous to $\mathrm{X}$-ray diffraction (XRD) in many respects. In principle, the intensity distribution by SLS and XRD both originate from the Bragg diffraction by the ordered lattice sites in crystal. The difference is the wavelength of the light source, which should be comparable with the interparticle distance. In the measurements of XRD, the light source is X-ray with very short wavelength. However, laser was used as light source in SLS because the interparticle distance in colloidal dispersions is in the range of visible light. For conventional SLS measurements, a mechanically rotated detector is used to collect the scattering light distribution at different scattering angle 
around the sample cell. Due to the rotation speed limitation of the detector, it needs several minutes to complete one measurement, which makes it difficult to measure crystal structures and other parameters rapidly. Therefore, SLS is mainly used in the study of steady state or the process in which the parameters of crystals change very slowly. Recently, with the developments of commercial equipments and some self-build setups, great improvements in time resolution had been achieved [26]. For example, in the method of SLS, CCD array was used to receive the scattering light in a rather wide range of scattering angle to get the intensity distribution. By this means, the mechanically rotated detector can be removed so that the sampling rate can be greatly increased. However, it is not convenient for CCD array to receive scattering light range from $0^{\circ}$ to $180^{\circ}$ mostly. So, some important data at certain scattering angle may be missed. For USAXS technique, the precise information including lattice parameters and crystal direction has been successfully obtained. But it is limited to the high contrast colloidal systems such as PMMA latex suspensions and silica dispersions. Sometimes a synchrotron source is needed for dispersions with low contrast [27].

In this study, we will demonstrate that a simple reflection spectrum method can achieve rapid and simultaneous measurement of important parameters of colloidal crystals by improving the analysis of the measured spectrum. Commonly, single diffraction peak is analyzed from the intensity-wavelength graph of RS. Instead, we analyzed multiple diffraction peaks from the intensitywave vector graph. By this means, more precise and quantitative information about the colloidal crystal could be obtained than previously reported $[11,13,14]$. Especially, the consistency of the results from RS and SLS presents convinced evidence that the crystal structure can be determined by the RS method alone. Therefore, the results clarified the misunderstanding that RS can not determine the crystal structure. Moreover, we prove that the RS method, as long as the relevant diffraction peaks are located in the available wavelength range, can be powerful for the researches of colloidal crystals, because lattice constant, crystal size and crystal structure etc. can all be simultaneously and rapidly measured. Besides, the determination of the crystal structure is much faster than conventional SLS, showing remarkable superiority of the RS method.

\section{Principle for the determination of crystal structure}

\subsection{Static light scattering}

SLS is one of the most commonly used tools to study the crystal structure and phase behaviors of colloidal crystal at present time. It is the same as XRD in principle, the difference is that laser with a certain wavelength is utilized instead of X-ray because of larger interparticle distance in colloidal dispersions. If we can collect the scattered light distribution at different angles and obtain the relationship between structure factor $S(q)$ and wave vector $q$ through next analysis, the crystal structure can be determined.

The wave vector can be expressed by the following equation:

$q=\left|q_{\mathrm{i}}-q_{\mathrm{s}}\right|=\frac{4 \pi n}{\lambda} \sin \left(\frac{\theta}{2}\right)$

where $q_{\mathrm{i}}$ and $q_{\mathrm{s}}$ are the wave-vectors of the incident and scattered light, respectively. $n$ is the refractive index of the suspending medium and $\lambda$ is the laser wavelength in vacuum. The measured sharp Bragg peaks from the crystallites could be observed at positions.

$q_{h k l}=\frac{2 \pi}{a} \sqrt{h^{2}+k^{2}+l^{2}}$

where $h, k, l$ are the Miller indices of the Bragg diffraction plane and $a$ is the lattice constant of the crystal.

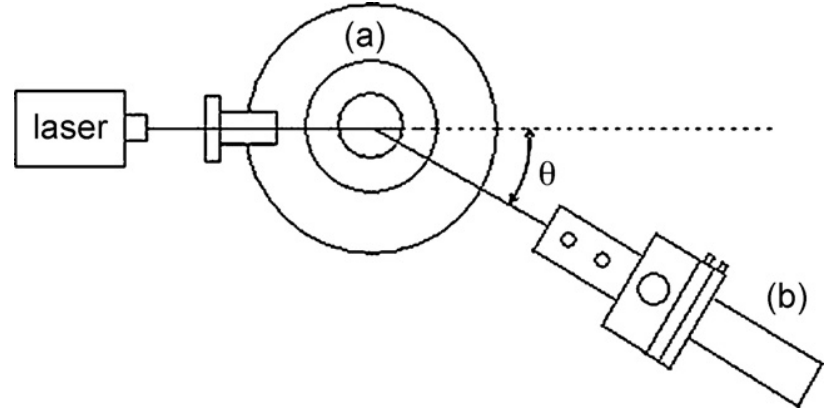

Fig. 1. Schematic diagram of the setup used in SLS: (a) sample cell and (b) detector.

A simple scheme of SLS is shown in Fig. 1. The detector is rotated around the sample to collect scattered light distribution at different angles, the structure factor $S(q)$ could be calculated from the measured scattered intensity $I(q)$ according to the following equation:

$I(q) \propto P(q) S(q)$

where $P(q)$ is the form factor of a single particle, it describes the angle dependence of single particles scattering and can be calculated theoretically or estimated experimentally on the dilute suspension in the presence of salt (with no crystallites). Experimentally, when the suspensions are very dilute, the scattered light intensity $I(q)$ can be used to substitute $S(q)$ [28-30]. In this paper, the colloidal suspensions used is dilute, so we use $I(q)$ instead of $S(q)$ in the later part.

\subsection{Reflection spectrum}

From the current opinion, reflection spectrum cannot be used to determine the crystal structure alone though it is widely used in the field of nucleation and growth kinetics $[11,13]$. However, if we can obtain the relationship between $I(q)$ and $q$ in a proper way from reflection spectrum, the structure of colloidal crystal will be able to be determined, just like XRD and SLS in principle.

According to Eq. (1), wave vector $q$ is the function of angle $\theta$ and wavelength $\lambda$, so the variation of $q$ can be realized through two approaches: one is by changing the scattered angle $\theta$; the other is by changing the wavelength $\lambda$ of the incident light.

In SLS, the scattered angle $\theta$ is variable and the wavelength $\lambda$ of the laser is fixed. On the contrary, the value of $\theta$ is fixed and the wavelength is variable for RS. The setup of RS used in this study is shown in Fig. 2. The light coming from light source hits the crys-

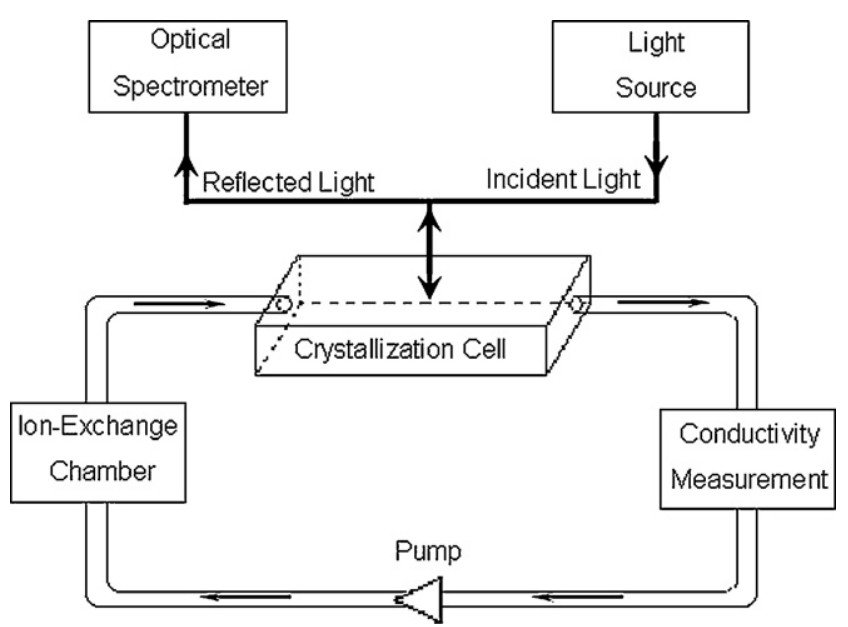

Fig. 2. Schematic diagram of the setup used in RS. The angle between the incident and reflected light is kept $180^{\circ}$. 
tallization cell perpendicularly, and the intensity of the reflected light is received by the spectrometer. The angle $\theta$ between the incident and reflected light is kept $180^{\circ}$ in the measurements. The halogen light source (Avalight-HAL, Avantes, Netherlands) can emit light with wavelength range from $300 \mathrm{~nm}$ to $1100 \mathrm{~nm}$. Through the changes of $\lambda$ we can also obtain $I(q)$ vs $q$, so reflection spectrum should also be able to determine the crystal structure.

In this paper, we will use SLS and RS respectively to analyze the structure and lattice constant of the crystal formed in the dilute colloidal dispersions. The validness of the reflection spectrum method to determine the structure is checked by comparing the experimental results from the two methods.

\section{Experiments}

\subsection{Sample preparation}

The charged polystyrene colloidal particles used in the experiments were synthesized by an emulsion polymerization method [31]. The as prepared PS latex was stored with resin (G501-X8(D), Bio-Rad Laboratories, USA) for further deionization. The diameter and polydispersity of the latex particles measured by dynamic light scattering method (Brookhaven Instruments Corporation, USA) were $102 \mathrm{~nm}$ and 0.05 , respectively. The surface charge density of latex particles is $11 \mu \mathrm{C} \mathrm{cm}^{-2}$ by conductometric titration. The suspensions used in the experiments were prepared by carefully weighting certain amount of PS latex, and then mixed it with double distilled water in a proper ratio. At last ultrasonic is used to obtain a homogeneous colloidal solution.

\subsection{Structural analysis of colloidal crystal}

The structural analysis of the colloidal crystal is based on the above mentioned methods, SLS and RS. The sketch diagrams of them are exhibited in Figs. 1 and 2.

In the measurement of SLS, some deionized suspensions were introduced into a cuvette with an inner diameter of $6 \mathrm{~mm}$. The cuvette stood vertically in a cylindrical vat which was filled with refractive index matched solvent and the temperature was kept at $25 \pm 0.1^{\circ} \mathrm{C}$. The light source is He-Ne laser with wavelength $532 \mathrm{~nm}$. The step angle of the detector is $0.2^{\circ}$ and the scanning range is $30-130^{\circ}$.

In the measurement of reflection spectrum, the colloidal suspensions were introduced into close circuit system consisted of tube, peristaltic pump, ion-exchange resin chamber, a conductivity measurement unit and crystallization cell, as shown in Fig. 2. The pump was used to circulate the suspension through ion-exchange resin and help the system stay in a state with low ion concentration. The crystallization process begins just after cessation of shear flow. The range of $q$ that can be provided by the spectrometer is about $17-50 \mu \mathrm{m}^{-1}$.

\section{Results and discussion}

The light intensity at different $q$ measured by SLS for steady colloidal crystals formed in suspension with volume fraction $0.3 \%$ was shown in Fig. 3. The wave vectors corresponding to the three Bragg peaks were $14.6 \mu \mathrm{m}^{-1}, 20.63 \mu \mathrm{m}^{-1}$ and $24.76 \mu \mathrm{m}^{-1}$, respectively. The ratio is $\sqrt{2}: \sqrt{4}: \sqrt{6}$. According to the Eq. (2) derived from selection rules, the crystal structure was identified to be body centered cubic (BCC) and the crystal planes were assigned to be (110), (200), and (211).

The reflection spectrum results for the same sample were displayed in Fig. 4. Fig. 4(A) is the typical RS pattern, in which the $x$-axis is the wavelength of the reflected light. Using Eq. (1), Fig. 4(A) can

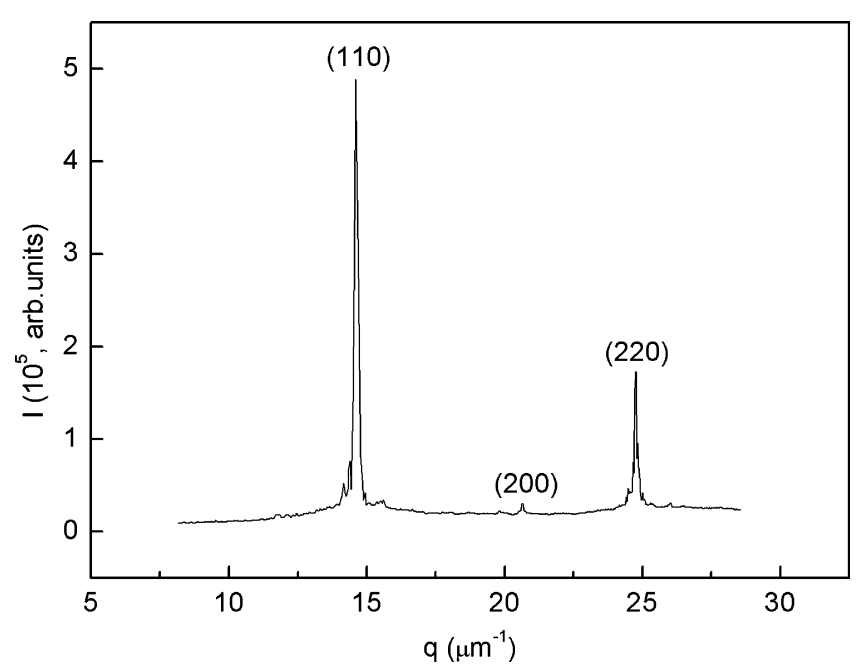

Fig. 3. SLS profile of the deionized polycrystalline PS sample ( $\Phi=0.3 \%$ ).

be converted to Fig. 4(B), in which the $x$-axis is the wave vector. It could be clearly seen that the converted pattern was very similar to the SLS profile (Fig. 3). They were both the light intensity as a function of wave vectors, i.e. the relationship of $I(q)$ and $q$. Then we can determine the crystal structure by the relative positions of the reflected peaks, just like the crystal structural analysis methodology used in SLS.

There are five reflected peaks in Fig. 4(B), the corresponding wave vectors were $20.34 \mu \mathrm{m}^{-1}, 24.84 \mu \mathrm{m}^{-1}, 28.59 \mu \mathrm{m}^{-1}$, $31.86 \mu \mathrm{m}^{-1}$ and $42.5 \mu \mathrm{m}^{-1}$. The ratio of them was $\sqrt{4}: \sqrt{6}: \sqrt{8}$ : $\sqrt{10}: \sqrt{18}$, so the crystal should be BCC and the reflected crystal planes were (200), (211), (220), (310) and (330). Meanwhile, we observed the higher order Bragg peaks (3 10) and (330) benefited from the wide range of wavelengths supplied by the light source.

Comparing Figs. 4(B) and 3, it can be seen that the determination of crystal structure by RS and SLS are quite similar in principle. Both of the methods need to know the ratio of the wave vectors corresponding to different peaks. Therefore, two or more peaks in the range of wave vectors are necessary for these methods to determine the crystal structure. It could be possible that, for some samples, only one peak or even no peak shows up in the wave vector range. In this condition, one should resort to some other structure characterization method.

In addition to structure determination, reflection spectrum can also obtain lattice constant value. It can be obviously seen from Eq. (2) that there should be a linear relationship between wave vector $q$ and $\left(h^{2}+k^{2}+l^{2}\right)^{1 / 2}$, and the slope is related to lattice constant. So, whether the linearity is good or not is a criterion to weight the validity of the new method. Look at Fig. $4(\mathrm{C})$ where $\left(h^{2}+k^{2}+l^{2}\right)^{1 / 2}$ was plotted as $q$, the linearity was very excellent. It indicated the method is indeed reasonable. The lattice constant obtained from the slope is $636 \mathrm{~nm}$ and the value calculated from the wave-vector $q$ of (2 20 ) is $621 \mathrm{~nm}$, showing good consistency. The nearest-neighbor interparticle distance in the bcc crystal can then be calculated to be about $538 \mathrm{~nm}$ (from the value of $a=621 \mathrm{~nm}$ ). However, the closest interparticle distance should be $622 \mathrm{~nm}\left(0.879 \mathrm{~d} \Phi^{-1 / 3}\right)$ if all the colloidal particles are forming uniform BCC symmetry. Therefore, the result provides a support to the viewpoint that sometimes attractive interparticle interaction must be considered in addition to purely repulsive forces [8-10].

In order to make a clear comparison, the results obtained from SLS and RS were both given in Table 1. Both of the two methods confirmed the crystal structure was BCC, the lattice constant val- 
Table 1

The comparison of SLS and RS results.

\begin{tabular}{|c|c|c|c|c|}
\hline Characterization method & Crystal structure & Lattice constant & $q$ value corresponding to (200) plane & $q$ value corresponding to (211) plane \\
\hline SLS & $\mathrm{BCC}$ & $637 \mathrm{~nm}$ & $20.63 \mu \mathrm{m}^{-1}$ & $24.76 \mu \mathrm{m}^{-1}$ \\
\hline RS & $\mathrm{BCC}$ & $636 \mathrm{~nm}$ & $20.34 \mu \mathrm{m}^{-1}$ & $24.84 \mu \mathrm{m}^{-1}$ \\
\hline
\end{tabular}
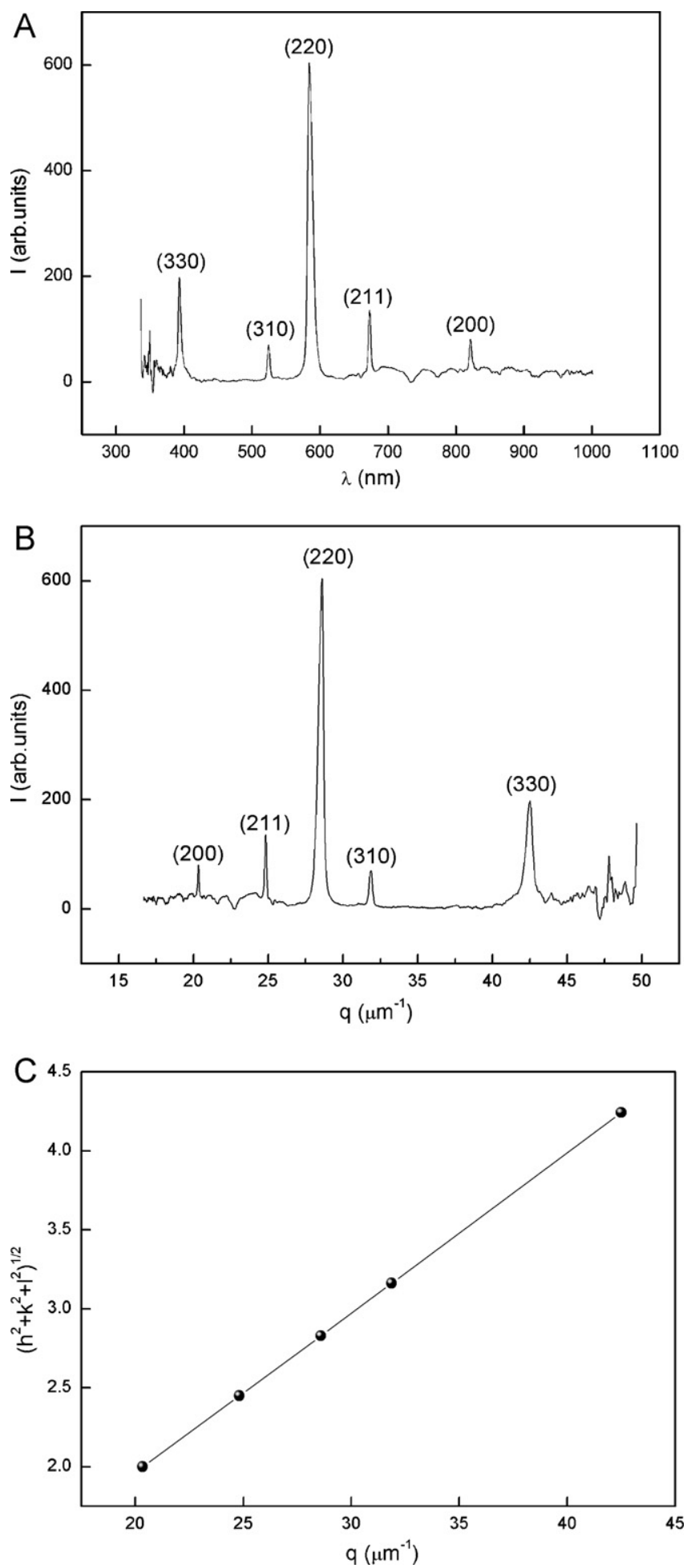

Fig. 4. The results of reflection spectrum measurement ( $\Phi=0.3 \%$ ): (A) $I$ vs $\lambda$, (B) $I$ vs $q$ and $(C)\left(h^{2}+k^{2}+l^{2}\right)^{1 / 2}$ vs $q$.

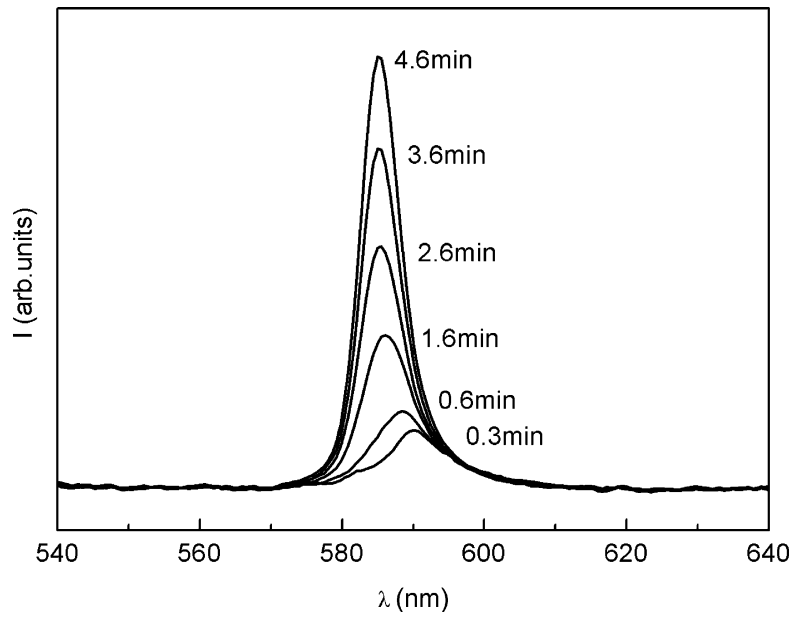

Fig. 5. Reflection spectra at different crystallization time for charged PS suspensions $(\Phi=0.3 \%)$.

ues were almost the same and the wave vectors corresponding to (2 000$),(211)$ is close too.

The changes of reflection spectrum patterns in the early growth stage of colloidal crystal $(\Phi=0.3 \%$ ) was shown in Fig. 5 . The reflection peak became sharp, and the peak intensity increased during crystallization, which corresponds to the increase in crystal size. As a matter of fact, not only the intensity but also the positions of the peaks changed with time. Under this rapid changing situation, conventional SLS will have some difficulties because of slow data collecting rate. RS may be a good complementary substitute. Benefited from the much faster sampling rate, reflection spectrum could determine the crystal structure promptly and it is very useful in monitoring the growth and possible structural changes during crystallization.

It can be seen that Fig. 4(B) (from RS) is very similar to Fig. 3 (from SLS). Therefore, we can conclude that the RS can substitute the conventional SLS method to a great extent for the study of colloidal crystals. Moreover, the RS method greatly increases the time resolution of the measurement comparing with conventional SLS. For SLS measurement, the consumed time was tens of minutes for scanning angle ranged from $0^{\circ}$ to $180^{\circ}$ with a step $0.2^{\circ}$. But in RS, the time resolution is greatly enhanced $(0.25 \mathrm{~s} / \mathrm{spectrum})$. It may be even more rapid with the upgrade of the instruments. So there is great potential application in studying the possible structural changes in the early stage of the crystallization process [32]. Therefore, we show that the lattice constant and crystal structure can both be simultaneously and rapidly measured by the RS method. Besides, some other parameters such as crystal size (actually the thickness) etc. can also be measured simultaneously and rapidly, as reported in some other studies [11,14].

\section{Conclusion}

Based on the principle analysis of conventional SLS method, we demonstrated that the reflection spectrum can also determine the structure of colloidal crystal as long as the relevant diffraction peaks located in the available wavelength range. The experimental results from RS are compared with those from conventional SLS method, 
showing excellent coincidence. The results clarified the long-time misunderstanding that the RS cannot be used to determine the crystal structure. The results also make RS method suitable for measuring the important parameters such as crystal structure, lattice constant and crystal size simultaneously and rapidly, making RS a more robust tool for the study of colloidal crystal. However, it should be noted that RS is not appropriate for very turbid samples, which is similar to other light scattering methods.

\section{Acknowledgment}

This work is supported by grant 10972217 from the National Natural Science Foundation of China and the Knowledge Innovation Program of the Chinese Academy of Sciences (including Grant No. KJCX2-YW-L08)

\section{References}

[1] B.V.R. Tata, S.S. Jena, Ordering, dynamics and phase transitions in charged colloids, Solid State Commun. 139 (2006) 562-580.

[2] J Yamanaka, H. Yoshida, T. Koga, N. Ise, T. Hashimoto, Reentrant solid-liquid transition in ionic colloidal dispersions by varying particle charge density, Phys. Rev. Lett. 80 (1998) 5806-5810.

[3] H. Yoshida, J. Yamanaka, T. Koga, T. Koga, N. Ise, T. Hashimoto, Transitions between ordered and disordered phases and their coexistence in dilute ionic colloidal dispersions, Langmuir 15 (1999) 2684-2702.

[4] P. Wette, I. Klassen, D. Holland-Moritz, D.M. Herlach, H.J. Schöpe, N. Lorenz, H. Reiber, T. Palberg, S.V. Roth, Communications: complete description of reentrant phase behavior in a charge variable colloidal model system, J. Chem. Phys. 132 (2010) 1311021-1311024.

[5] T. Okubo, Phase transition between liquid-like and crystal-like structures deionized colloidal suspensions, J. Chem. Soc., Faraday Trans. 86 (1990) 2871-2876.

[6] Y. Monovoukas, A.P. Gast, The experimental phase diagram of charged colloidal suspensions, J. Colloid Interface Sci. 128 (1989) 533-548.

[7] E.B. Sirota, H.D. Ou-Yang, S.K. Sinha, P.M. Chaikin, J.D. Axe, Y. Fujii, Complete phase diagram of a charged colloidal system: a synchrotron X-ray scattering study, Phys. Rev. Lett. 62 (1989) 1524-1527.

[8] N. Ise, T. Konishi, B.V.R. Tata, How homogeneous are "homogeneous dispersions"? Counterion-mediated attraction between like-charged species, Langmuir 15 (1999) 4176-4184.

[9] P.S. Mohanty, B.V.R. Tata, A. Toyotama, T. Sawada, Gas-solid coexistence in highly charged colloidal suspensions, Langmuir 21 (2005) 1167811683.

[10] B.V.R. Tata, P.S. Mohanty, M.C. Valsakumar, Bound pairs: direct evidence for long-range attraction between like-charged colloids, Solid State Commun. 147 (2008) 360-365.

[11] T. Okubo, A. Tsuchida, T. Kato, Nucleation and growth processes in the colloidal crystallization of silica spheres in the presence of sodium chloride as studied by reflection spectroscopy, Colloid Polym. Sci. 277 (1999) 191-196.
[12] T. Okubo, H. Hase, H. Kimura, E. Kokufuta, Thermosensitive colloidal crystals of silica spheres in the presence of gel spheres of poly( $\mathrm{N}$-isopropyl acrylamide), Langmuir 18 (2002) 6783-6788.

[13] T. Okubo, H. Ishiki, Kinetic analyses of colloidal crystallization in a wide range of sphere concentrations as studied by reflection spectroscopy, J. Colloid Interface Sci. 228 (2000) 151-156.

[14] T. Okubo, Ordered solution structure of a monodispersed polystyrene latex as studied by the reflection spectrum method, J. Chem. Soc., Faraday Trans. 82 (1986) 3163-3173.

[15] T. Yoshiyama, I. Sogami, N. Ise, Kossel line analysis on colloidal crystals in semidilute aqueous solutions, Phys. Rev. Lett. 53 (1984) 2153-2156.

[16] T. Shinohara, T. Kurokawa, T. Yoshiyama, T. Itoh, I.S. Sogami, N. Ise, Structure of colloidal crystals in sedimenting mixed dispersions of latex and silica particles, Phys. Rev. E 70 (2004) 0624011-0624014.

[17] L. Liu, S.H. Xu, J. Liu, Z.W. Sun, Characterization of crystal structure in binary mixtures of latex globules, J. Colloid Interface Sci. 326 (2008) 261-266.

[18] P. Wette, H.J. Schöpe, J. Liu, T. Palberg, Characterization of colloidal solids, Prog. Colloid Polym. Sci. 123 (2004) 264-268.

[19] J.K.G. Dhont, C. Smits, H.N.W. Lekkerkerker, A time resolved static light scattering study on nucleation and crystallization in a colloidal system, J. Colloid Interface Sci. 152 (1992) 386-401.

[20] T. Okubo, K. Kiriyama, N. Nemoto, H. Hashimoto, Static and dynamic lightscattering of colloidal gases, liquids and crystals, Colloid Polym. Sci. 274 (1996) 93-104.

[21] P.S. Mohanty, W. Richtering, Structural ordering and phase behavior of charged microgels, J. Phys. Chem. B 112 (2008) 14692-14697.

[22] H. Matsuoka, K. Kakigami, N. Ise, Y. Kobayashi, Y. Machitani, T. Kikuchi, T. Kato Ultra-small-angle X-ray-scattering study: preliminary experiments in colloidal suspensions, PNAS 88 (1991) 6618-6619.

[23] T. Konishi, N. Ise, H. Matsuoka, H. Yamaoka, I.S. Sogami, T. Yoshiyama, Structural study of silica particle dispersions by ultra-small-angle X-ray scattering, Phys. Rev. B 51 (1995) 3914-3917.

[24] T. Konishi, N. Ise, Single crystal of colloidal silica particles in a dilute aqueous dispersion as studied by a two-dimensional ultrasmall-angle X-ray scattering Phys. Rev. B 57 (1998) 2655-2658.

[25] W.L. Vos, M. Megens, C.M. van Kats, P. Bösecke, X-ray diffraction of photonic colloidal single crystals, Langmuir 13 (1997) 6004-6008.

[26] P. Wette, A. Engelbrecht, R. Salh, I. Klassen, D. Menke, D.M. Herlach, S.V. Roth, H.J. Schöpe, Competition between heterogeneous and homogeneous nucleation near a flat wall, J. Phys.: Condens. Matter 21 (2009) 4641151-46411510.

[27] T. Harada, H. Matsuoka, Ultra-small-angle X-ray and neutron scattering study of colloidal dispersions, Curr. Opin. Colloid Interface Sci. 8 (2004) 501-506.

[28] A. Guinier, G. Fournet, in: C.B. Walker (Ed.), Small-angle scattering of X-rays, John Wiley and Sons, Inc., New York, 1955.

[29] H. Matsuoka, H. Murai, N. Ise, "Ordered" structure in colloidal silica particle suspensions as studied by small-angle X-ray scattering, Phys. Rev. B 37 (1988) 1368-1375.

[30] H.J. Schöpe, T. Palberg, A multipurpose instrument to measure the vitreous properties of charged colloidal solids, J. Colloid Interface Sci. 234 (2001) 149-161.

[31] G.T.D. Shouldice, G.A. Vandezande, A. Rudin, Practical aspects of the emulsifierfree emulsion polymerization of styrene, Eur. Polym. J. 30 (1994) 179-183.

[32] S. Xu, H. Zhou, Z. Sun, J. Xie, Formation of an fcc phase through a bcc metastable state in crystallization of charged colloidal particles, Phys. Rev. E 82 (2010) 010401. 